\title{
Multiple clinical episodes of Plasmodium falciparum malaria in a low transmission intensity setting: exposure versus immunity
}

Josea Rono ${ }^{1,2}$, Anna Färnert², Linda Murungi', John Ojal', Gathoni Kamuyu', Fatuma Guleid ${ }^{1}$, George Nyangweso', Juliana Wambua ${ }^{1}$, Barnes Kitsao ${ }^{1}$, Ally Olotu' ${ }^{1}$ Kevin Marsh ${ }^{1,3}$ and Faith HA Osier ${ }^{1 *}$

\begin{abstract}
Background: Epidemiological studies indicate that some children experience many more episodes of clinical malaria than their age mates in a given location. Whether this is as a result of the micro-heterogeneity of malaria transmission with some children effectively getting more exposure to infectious mosquitoes than others, or reflects a failure in the acquisition of immunity needs to be elucidated. Here, we investigated the determinants of increased susceptibility to clinical malaria by comparing the intensity of exposure to Plasmodium falciparum and the acquisition of immunity in children at the extreme ends of the over-dispersed distribution of the incidence of clinical malaria.
\end{abstract}

Methods: The study was nested within a larger cohort in an area where the intensity of malaria transmission was low. We identified children who over a five-year period experienced 5 to 16 clinical malaria episodes (children at the tail-end of the over-dispersed distribution, $n=35)$, remained malaria-free $(n=12)$ or had a single episode $(n=26)$. We quantified antibodies against seven Plasmodium falciparum merozoite antigens in plasma obtained at six cross-sectional surveys spanning these five years. We analyzed the antibody responses to identify temporal dynamics that associate with disease susceptibility.

Results: Children experiencing multiple episodes of malaria were more likely to be parasite positive by microscopy at cross-sectional surveys ( $X^{2}$ test for trend $14.72 P=0.001$ ) and had a significantly higher malaria exposure index, than those in the malaria-free or single episode groups (Kruskal-Wallis test $P=0.009$ ). In contrast, the five-year temporal dynamics of anti-merozoite antibodies were similar in the three groups. Importantly in all groups, antibody levels were below the threshold concentrations previously observed to be correlated with protective immunity.

Conclusions: We conclude that in the context of a low malaria transmission setting, susceptibility to clinical malaria is not accounted for by anti-merozoite antibodies but appears to be a consequence of increased parasite exposure. We hypothesize that intensive exposure is a prerequisite for protective antibody concentrations, while little to modest exposure may manifest as multiple clinical infections with low levels of antibodies. These findings have implications for interventions that effectively lower malaria transmission intensity.

Keywords: Clinical malaria, Immunity, Over-dispersion, Antibodies, Merozoite, Plasmodium falciparum

\footnotetext{
* Correspondence: FOsier@kemri-wellcome.org

'KEMRI-Wellcome Trust Research Programme, Centre for Geographical

Medicine Research-Coast, Kilifi, Kenya

Full list of author information is available at the end of the article
} 


\section{Background}

Heterogeneity in the risk of Plasmodium falciparum malaria in malaria-endemic areas has long been recognized as a common feature of the epidemiology of malaria [1]. Recently, this phenomenon has been described by studies in Senegal [2], Uganda [3] and Kenya [4,5] as well as in large datasets drawn from 90 populations in Africa [6]. In Senegal a subset of children experienced up to twenty malaria episodes in their first two years of life while their age- and location-mates experienced only one episode over the same period [2]. Analysis of the distribution of malaria in a longitudinally monitored population in Kenya revealed that the incidence of malaria was heterogeneous and followed a negative binomial distribution, a phenomenon that was described as overdispersion [5]. Heterogeneity in infection burden is also evident in other infectious diseases where a small proportion (approximately 20\%) of the population is intensely infected and responsible for about $80 \%$ of the infectious agent's transmission, an observation referred to as the '20/80' rule [7].

The factors underlying the heterogeneous epidemiology of malaria are not fully understood. The heterogeneity has been partly attributed to differences in: human genetic [3] and behavioral [8] factors, distance to mosquito breeding grounds $[3,9,10]$, household-related factors [9] and human-mosquito interactions [11]. However, whether children at the tail end of the overdispersed distribution of malaria differ from children experiencing fewer malaria attacks in their ability to acquire immunity to malaria, as assessed by antibody responses to $P$. falciparum antigens is unknown.

Here, we describe the temporal dynamics of antimerozoite antibodies in children who were part of the Kenyan cohort described above [5] and differing in their incidence of malaria to determine whether failure to acquire antibodies against these antigens may explain the differences in susceptibility to malaria. We identified, within this cohort and during a five-year follow up period, children who: experienced 5 to 16 episodes of clinical malaria (children at the tail end of the overdispersed distribution and hereafter referred to as the 'multiple-episodes' group), did not experience clinical malaria ('malaria-free' group) or had only one episode of clinical malaria ('single-episode' group). We then measured antibodies to seven merozoite antigens in these children at six cross-sectional surveys spanning the fiveyear period and compared the temporal dynamics of anti-merozoite antibodies.

\section{Methods}

\section{Study population}

The study was conducted within a longitudinally monitored population in Ngerenya, located within Kilifi District at the Kenyan coast [5,12]. This population has been monitored from 1998 to date. During this time parasite prevalence declined dramatically such that by 2009 parasite prevalence was zero and has remained so since (Additional file 1: Figure S1). The present report focuses on a subset of children (Figure 1) who were 0.5to 3-years old in September 1998 (and 5.5- to 8-years old in October 2003) so as to capture the period during which considerable buildup of naturally-acquired antimerozoite antibodies has been observed in this cohort [13]. During this period there was active weekly surveillance of the cohort and malaria episodes were recorded by active and passive case detection [12]. At the weekly visits children were tested for malaria parasites only if they were symptomatic and treated if parasitemic. In the present analysis, a case of clinical malaria was defined as fever (axillary temperature $\geq 37.5^{\circ} \mathrm{C}$ ) and any level of parasitemia for children $<1$-year old and fever accompanied by parasitemia of $\geq 2,500$ parasites/ $\mu$ l of blood for children $\geq 1$-year old [12]. During the same period, six cross-sectional surveys (in September 1998, October 2000, May 2002, October 2002, May 2002 and October 2003) were conducted before the high malaria transmission seasons at which venous blood was collected, and plasma and packed cells stored. At each survey, thick and thin blood smears were prepared and axillary temperature recorded for all participants. Children who were parasitemic at the surveys were not treated for malaria unless they were also symptomatic. Ethical approval for this study was obtained from the KEMRI National Ethics Committee and written informed consent was obtained from the guardians of all children. We compared antibody levels in this cohort (the October 2000 sample) to those in other cohorts we have previously studied [14,15]. Ethical approval for this latter analysis was not required.

\section{Determination of the malaria exposure index}

The malaria exposure index estimates a distanceweighted local prevalence of malaria infection within a kilometer radius around an index child [16]. In essence, an individual's level of exposure is inferred not from their own history, but from that of the children surrounding them. Children with a high exposure index are more likely to be surrounded by malaria infected children, while those with a low exposure index are not. The malaria exposure index had previously been calculated for the children included in this study [16].

\section{Detection of $P$. falciparum by microscopy}

The detection of $P$. falciparum in whole blood samples collected at the cross-sectional surveys has been described previously [12]. Briefly, thick and thin blood 


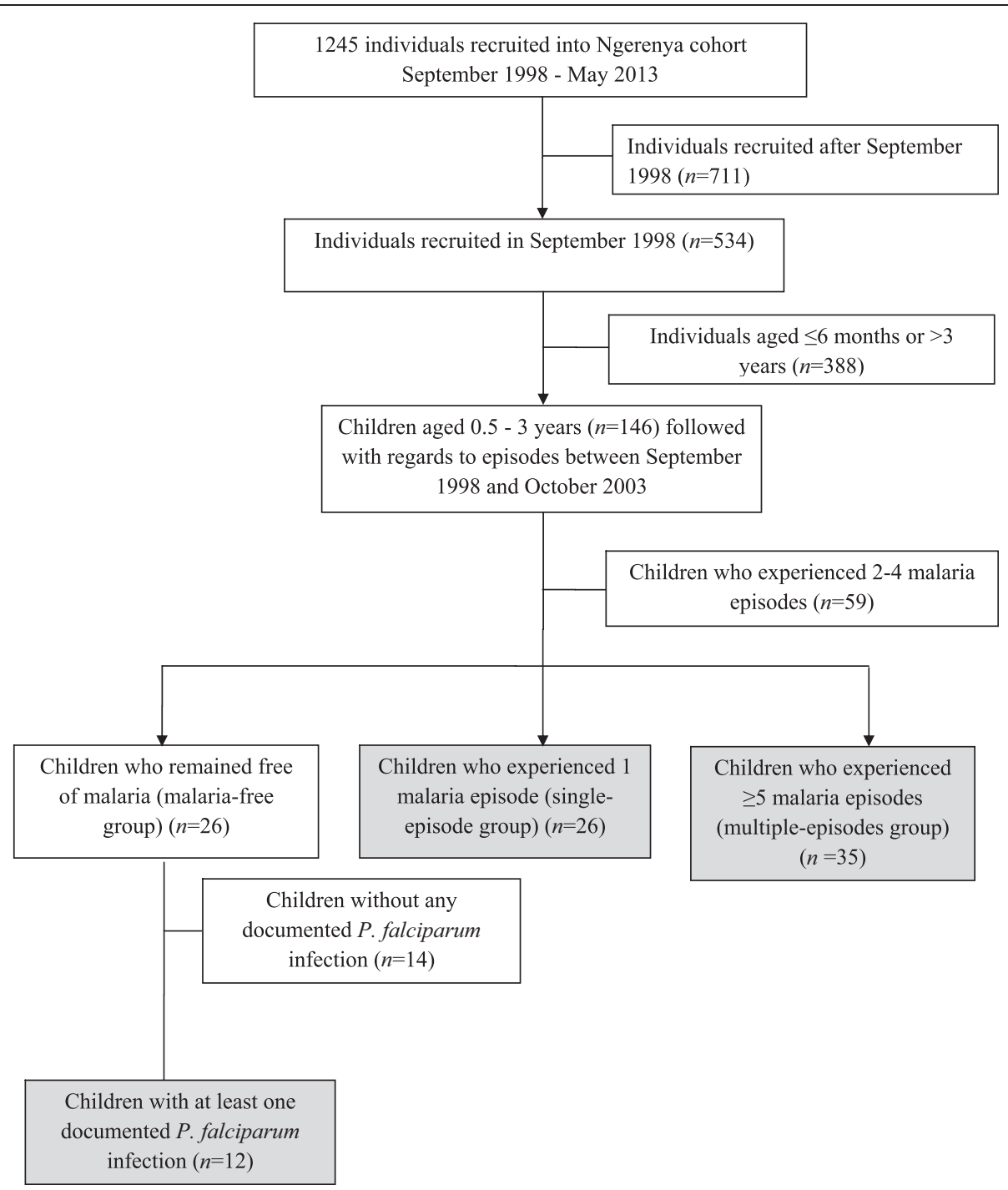

Figure 1 Inclusion of children into the malaria-free, single-episode and multiple-episodes groups. The gray-shaded boxes indicate the number of children included in the three groups investigated in this study.

slides were examined by microscopy and parasite densities determined as the number of parasites per 8,000 white blood cells $/ \mu \mathrm{L}$ of blood.

\section{Genotyping of $P$. falciparum infections}

Genomic DNA was extracted from packed erythrocytes using QiaAmp Blood Mini kit (Qiagen, Crawley, UK). Genotyping of $P$. falciparum $m s p 2$ gene was performed as previously described [17]. Briefly, the PCR included an initial amplification of the outer $m s p 2$ domain, followed by nested reactions with fluorescently labeled primers targeting the FC27 and IC-1/3D7allelic types of $m s p 2$. Fragment sizes were determined by capillary electrophoresis and analyzed using GeneMapper software (Applied Biosystems).

\section{Recombinant $P$. falciparum merozoite antigens}

Five recombinant antigens representing four vaccine candidate antigens were expressed in Escherichia coli. The 19-kilodalton fragment of merozoite surface protein (MSP) one-1 (MSP-1 19 ) [18], P. falciparum reticulocytebinding homologue 2 (PfRh2) [19], and two allelic forms of MSP-2: MSP-2_Dd2 (corresponding to the FC27 $m s p 2$ allelic family) and MSP-2_CH150/9 (corresponding to the IC-1 $m s p 2$ allelic family) [20] were expressed as glutathione-S-transferase-fusion proteins. Recombinant MSP-3_3D7 antigen was expressed as a maltosebinding protein-fusion protein [21]. Apical merozoite antigen 1 (AMA-1) from the 3D7 and FVO strains was expressed in Pichia pastoris as 6xHis-fusion proteins [22] and generously provided by Dr. Edmond Remarque. 
Apart from PfRh2 and MSP-1 19 , all antigens were expressed as full-length proteins.

\section{Multiplex bead-based antibody assay}

Plasma immunoglobulin G (IgG) to the recombinant antigens was measured using a previously described multiplex bead-based assay [15]. Serially-diluted malariaimmune globulin (MIG) [23] was included in each plate as a standard positive control, allowing for the conversion of mean fluorescent intensities to relative antibody concentrations in arbitrary units (AUs) and correction of inter-plate variation. Negative controls, consisting of pooled plasma from adult $P$. falciparum unexposed donors residing in the United Kingdom, were included in each plate to allow for the determination of seropositivity cut-offs. The seropositivity cut-off was determined as the mean fluorescent intensity (MFI) of the negative control plus two standard deviations.

\section{Data analysis}

Data analysis was performed using STATA 11.2. Antibody titers measured in this study were compared to threshold antibody concentrations. These are antibody concentrations against individual antigens measured that appeared to be associated with protection against clinical episodes of malaria in two independent cohort studies $[14,15]$, and were calculated using a standard reference reagent. The threshold antibody concentrations for antibody responses to MSP-1 19 , MSP-2, MSP-3_3D7 and AMA-1 antigens were 59, 19, 16 and 55 AUs, respectively $[14,15]$. Antibody titers in children included in this study were also compared to age-matched children in two independent cohorts: the Chonyi cohort in Kenya with $P f \mathrm{PR}_{2-10}$ of $44 \%$ [14] and a cohort in Rufiji District, Tanzania with $P f \mathrm{PR}_{2-10}$ of $49 \%[15,24]$. The ${\mathrm{P} f R_{2-10}}$ is a measure of malaria transmission intensity at a population level [25-28].

Differences in rates of change in antibody titers over the study period were tested using a multilevel mixedeffects linear regression model that accounts for inherent correlations between repeated measurements done on the same subject [29]. In this model, differences in the rates of change of antibody titers in the singleepisode and multiple-episodes groups were estimated relative to the malaria-free group and reported as coefficients. The model also took into account the presence or absence of asymptomatic parasitemia at each crosssectional survey and the number of times an individual was parasitemic during the five year longitudinal follow up.

\section{Results}

In total 1,245 individuals were recruited into the Ngerenya cohort between September 1998 and May 2013. The present study was restricted to the follow up time between September 1998 and October 2003 because this was a five-year period when, compared to other years, P. falciparum transmission was highest (Additional file 1: Figure S1). In September 1998 the cohort included 534 individuals (1-month to 82-years old) of which 146 were children 0.5- to 3-years old (Figure 1). Out of the 146 children, 26 had no record of clinical malaria between September 1998 and October 2003; of these 12 had at least one documented asymptomatic $P$. falciparum infection as determined by microscopy (either at crosssectional surveys or weekly follow up visits) and were classified into the malaria-free group (Figure 1). The remaining 14 were not studied further as exposure to $P$. falciparum parasites could not be definitively ascertained. Twenty six children experienced one episode of malaria in the five-year follow up period and were classified into the single-episode group (Figure 1) while 35 children experienced five to sixteen malaria episodes (Figure 2) and were classified into the multiple-episodes group (Figure 1). The average incidence of malaria was 0.2 and 1.59 (range: 1 to 3.2) episodes/person/year in the single-episode and multiple-episodes groups, respectively. Children who experienced two to four episodes of malaria in the five years of follow up $(n=59)$ were excluded from the present analysis so as to allow for a comparison of antibody responses in children at the extreme ends of the over-dispersed clinical malaria incidence distribution (Figure 1). The age profiles of the study groups were comparable (Table 1).

\section{Distribution of exposure to Plasmodium falciparum parasites in the three study groups}

The proportion of surveys at which children were parasitemic by microscopy was larger in the multipleepisodes group compared to the single-episode and malaria-free groups of children $\left(X^{2}\right.$ test for trend 14.72 $P=0.001$, Table 1). Children in the multiple-episodes group had higher malaria exposure, as measured by the malaria exposure index (a distance-weighted local prevalence of malaria) [16], compared to children in the single-episode and malaria-free groups (Kruskal-Wallis test $P=0.009$, Table 1). The overall PfPR $2-10$ in the Ngerenya cohort decreased over the study period with the decrease being more evident in the malaria-free and single-episode groups of children compared to the multiple-episodes group (Figure 3). P. falciparum parasites were detected by PCR in $26.7 \%, 19.5 \%$ and $24.4 \%$ of the children at the September 1998, October 2000 and May 2002 cross-sectional surveys, respectively. The number of $m s p 2$ genotypes, that is, clones in PCR positive samples at the cross-sectional surveys, ranged from one to four, and was higher in the multipleepisodes group than in the single-episode and malaria-free groups (Additional file 1: Figure S2). 


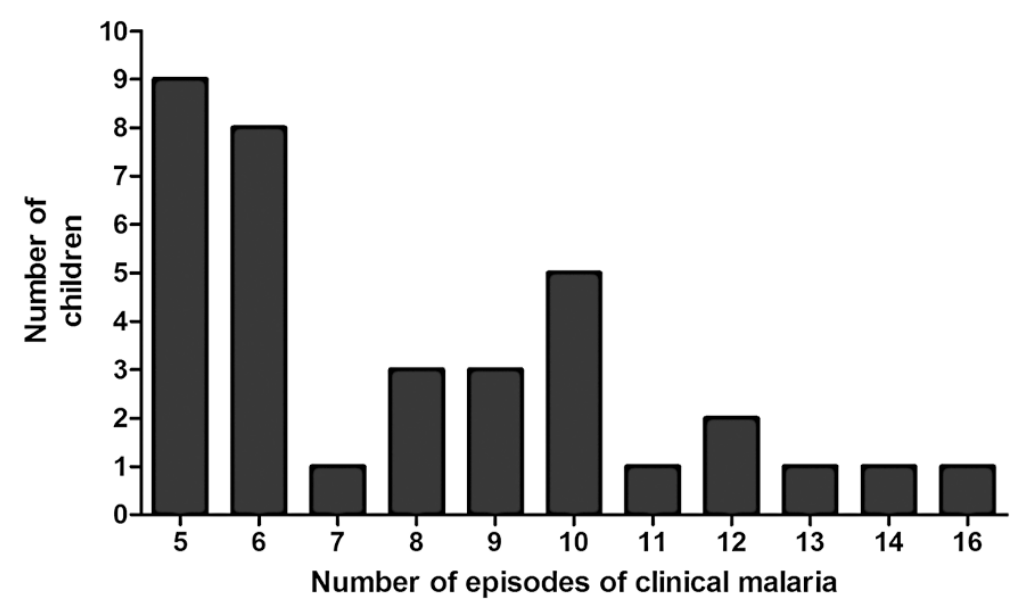

Figure 2 Distribution of clinical malaria episodes per child among children in the multiple-episodes group. The histogram shows the number of children (y axis) within the multiple-episodes group with a given number of clinical malaria episodes (x axis) between September 1998 and October 2003.

Antibody profiles of individual children over time

Diverse longitudinal antibody profiles were observed in individual children across the three groups (Figure 4). Some children maintained relatively low antibody titers to all the antigens and only had relatively high titers at those cross-sectional surveys at which they were parasitemic (Figure 4A, E, G, H and I). In children with these profiles, there was an indication of allele-specific boosting of anti-MSP-2 antibody responses; infections with clones of IC-1 or FC $m s p 2$ types were associated with high antibody titers to the corresponding MSP-2 type antigen (Figure 4A, F and H). For instance, the IC-1 infection in May 2002 in child N0102 (Figure 4I) coincides with high titers to MSP-2_Ch150/9 but not to MSP-2_Dd2. Some children, however, had profiles characterized by relatively high antibody titers to some antigens at surveys at which they were aparasitemic (Figure 4B). Remarkably, some children maintained low antibody titers despite having a high intensity of asymptomatic infections and episodes of malaria (Figure $4 \mathrm{~F}$ and $\mathrm{H}$ ).

\section{Comparison of antibody titers at individual cross-sectional} surveys in the three study groups

Comparisons of antibody titers to each of the seven antigens in the three study groups are shown in Figure 5A-5G. Generally, the multiple-episodes group had lower titers to MSP-1 $_{19}$ (Figure 5A) but higher titers to MSP-2_Dd2 (Figure 5B), MSP-3_3D7 (Figure 5D), and both AMA-1 alleles (Figure 5E and F) compared to the single-episode and malaria-free groups. To better understand the antibody data, we compared antibody titers to MSP-1 $1_{19}$, MSP-2 and AMA-1 in the three study groups to titers in age-matched children in the Chonyi [14] and Tanzania [15] cohorts. Antibody titers in children included in this study were generally lower than in age-matched children in the Chonyi and Tanzania cohorts (Additional file 1: Figure S3) and were also below threshold concentrations that appear to be necessary for protection against clinical episodes of malaria $[14,15]$. Moreover, antibody titers obtained with a pool of hyperimmune sera (PHIS) were well above these threshold concentrations for all the antigens tested (Figure 5).

Table 1 Baseline characteristics

\begin{tabular}{|c|c|c|c|}
\hline \multirow[b]{2}{*}{ Characteristic } & \multicolumn{3}{|l|}{ Group of children } \\
\hline & Malaria-free & Single-episode & Multiple-episodes \\
\hline Number & 12 & 26 & 35 \\
\hline Age, median age in years (IQR) & $1.67(1.36$ to 2.12$)$ & $1.52(1.21$ to 2.21$)$ & $1.78(1.38$ to 2.41$)$ \\
\hline Sex, number female (\%) & $4 / 12(33.33)$ & $8 / 26(30.77)$ & $15 / 35(42.86)$ \\
\hline Hemoglobin AS, number (\%) & $3(27.27)$ & $4(17.39)$ & $1(3.13)$ \\
\hline $\begin{array}{l}\text { Proportion of cross-sectional surveys at which children were parasite positive by } \\
\text { microscopy, proportion (\%) }\end{array}$ & 7.5 & 8 & 24.26 \\
\hline Malaria exposure index, median $(\mathrm{IQR})^{\mathrm{C}}$ & 0.39 (0.31 to 0.50$)$ & $0.54(0.47$ to 0.73$)$ & $0.65(0.52$ to 0.76$)$ \\
\hline
\end{tabular}

${ }^{\mathrm{a}}$ Data available for 11, 23 and 32 children in the malaria-free, single-episode and multiple-episodes groups respectively. ${ }^{\mathrm{b}}$ There was a non-significant trend towards a larger proportion of children in the malaria-free group compared to single-episode and multiple-episodes groups having the sickle cell trait (Fisher's exact test $P=0.054)$. ${ }^{C}$ Data available for 10,21 and 24 children in the malaria-free single-episode and multiple-episodes groups respectively. Age is at the first sampling point in September 1998. IQR, interquartile range. 


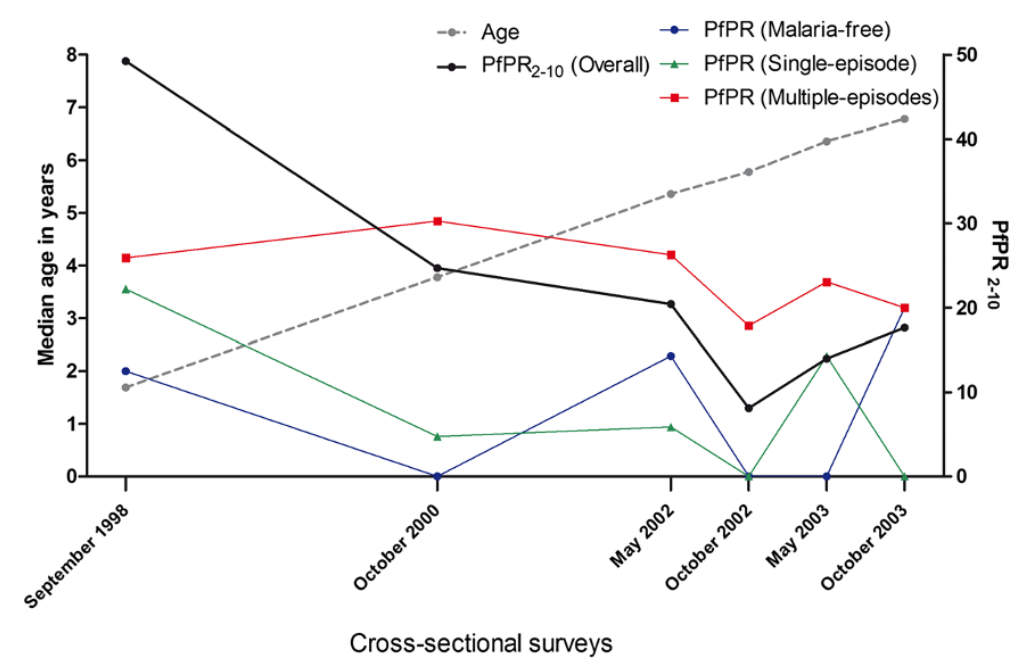

Figure 3 Temporal change in age and parasite prevalence during the study period. The plot shows the median age in years (left y axis) of the children included in this study, the parasite prevalence rates in malaria-free (blue circles), single-episode (green triangles) and multiple-episodes (red squares) groups of children as well as the overall parasite prevalence (black circles) in children 2- to 10-years old (PfPR 2 -10) in the entire Ngerenya cohort at the six cross-sectional surveys.

\section{Comparisons of the temporal changes in antibody response in the three study groups}

At the start of this study (September 1998), antibody titers to all antigens were comparable among the three study groups. Univariate analysis showed evidence for a significantly higher rate of increase in antibody titers to AMA-1_FVO and AMA_3D7 over the five-year period in the multiple-episodes compared to the single-episode group of children (Table 2). After adjusting for both sickle cell trait and asymptomatic parasitemia, the difference in the rate of increase in antibody titers was only evident for AMA-1_FVO (coefficient -6.32, 95\% confidence interval (CI) -12.64 to -0.01 , Table 2). There were no significant differences in rates of change of antibody titers to other merozoite antigens among the three study groups (Table 2).

\section{Discussion}

This is, to our knowledge, the first attempt to investigate the underlying immunological determinants of the overdispersion of clinical episodes of malaria in a low transmission intensity setting. Our study showed that over a five-year period, children who were susceptible to multiple clinical attacks of malaria had higher indices of exposure to infectious mosquitoes than those with single or no episodes. In contrast, the temporal dynamics of antibody responses to Plasmodium falciparum merozoite antigens were generally similar in all groups of children. Of note, antibody levels in all groups of children were lower than those previously shown to correlate with protection against clinical malaria. These data suggest that the differences in susceptibility to clinical malaria among children in this context are attributable to differences in exposure to infectious mosquitoes rather than to a failure to acquire immunity, as reflected by anti-merozoite antibodies.

The malaria exposure index [16] estimates a distanceweighted local prevalence of malaria infection within a kilometer radius around an index child. By this measure, children in the multiple-episodes group had more exposure to the parasite compared to children in the malariafree and single-episode groups. Coupled with the fact that the children in the multiple episode group were also more likely to be parasite positive (asymptomatically parasitemic) and with more clones (parasites of different msp2 genotypes) at the cross-sectional surveys is a strong indication that children in the multiple episodes groups are indeed more exposed to the parasite. In the absence of clear differences in the acquisition of immunity (as estimated by anti-merozoite antibodies), our data suggest that the differences in disease susceptibility were driven by the observed differences in the intensity of exposure to the parasite.

Malaria transmission intensity was low in the study area. P. falciparum exposure (as estimated by PfPR) in the three groups of children studied here did not exceed $30 \%$ at any of the cross-sectional surveys over five years. This is relatively low compared to the $40 \%$ cutoff above which a population is considered to be under high malaria transmission [25], and is supported by our finding that antibody titers in these children were lower than those of age-matched children in separate cohorts under higher malaria transmission intensity. Furthermore, the incidence rate of malaria in the multiple-episodes group 


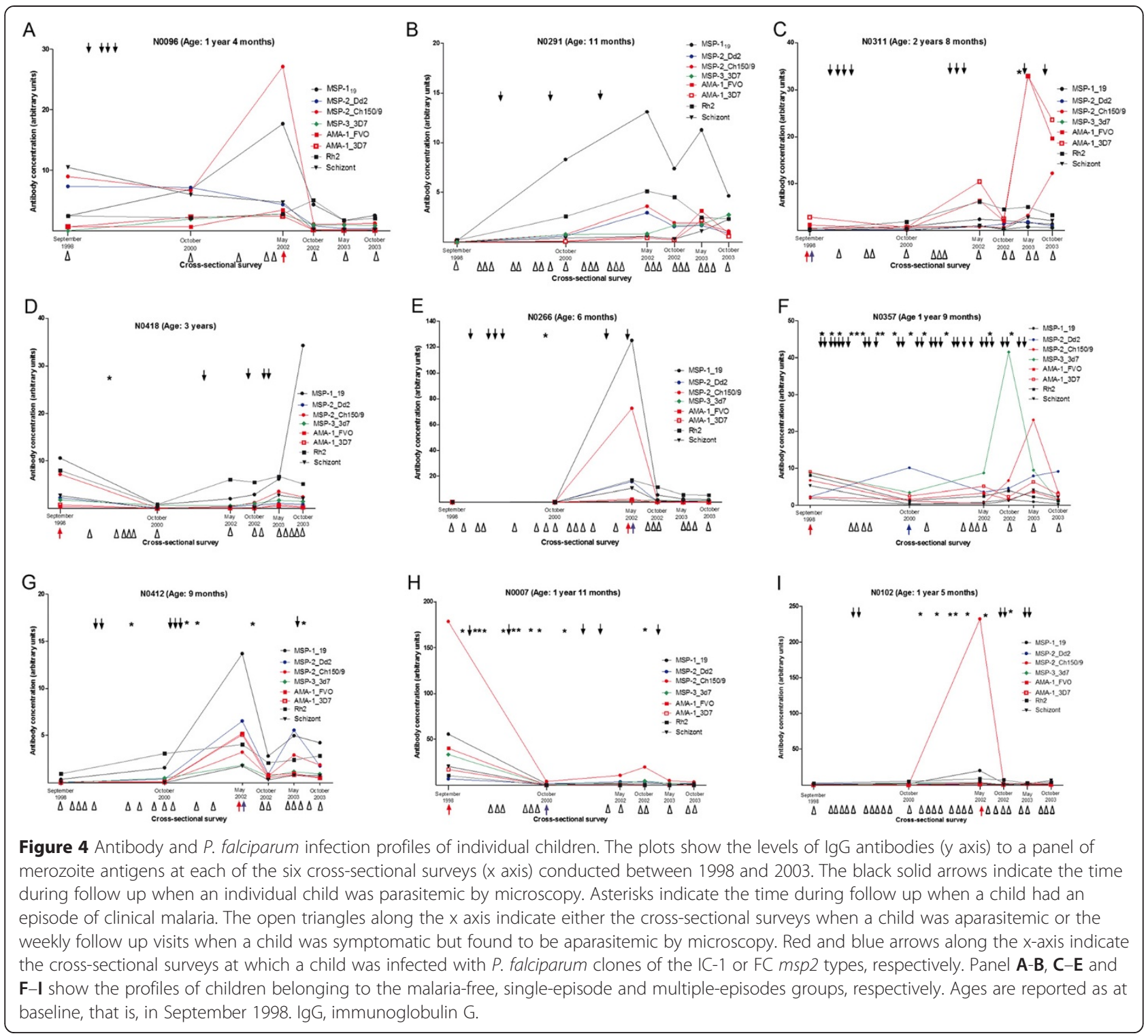

(1.59 episodes/person/year) is less than a third of what has been reported from high malaria transmission areas (5.3 episodes/person/year) [30]. The low intensity of malaria transmission probably accounts for the fact that although children in the multiple-episodes group had higher indices of parasite exposure, their antibody levels were still lower than those known to correlate with protection against clinical episodes of malaria $[14,15]$. It may also explain why no significant differences in antibody levels were observed between the multiple episodes group and the malaria-free or single-episode groups.

Overall, with the exception of AMA-1-FVO, the rate of change of antibody titers over time did not differ significantly between the three groups. The absence of any significant differences in the temporal change in antibody titers between the groups could be attributable to the young age of the study subjects, small sample size or the antigens studied. It is possible that the young age of the study subjects precludes any potential differences in rates of buildup of antibodies among the study groups. This is plausible considering that, given uniform parasite exposure, the buildup of antibody titers is slower in children than in adults [31], implying that young age is inherently associated with slow acquisition of antibody responses. The present study was limited to a panel of merozoite antigens to which antibody titers have been shown to correlate with protective immunity [32]. The selection of these antigens allowed for the use of antibody titers as proxies of naturally acquired immunity.

Generally, we observed allele-specific boosting of antiMSP-2 antibodies, which has also been reported in relation to MSP-2 [33] and AMA-1 [34] and suggests that 

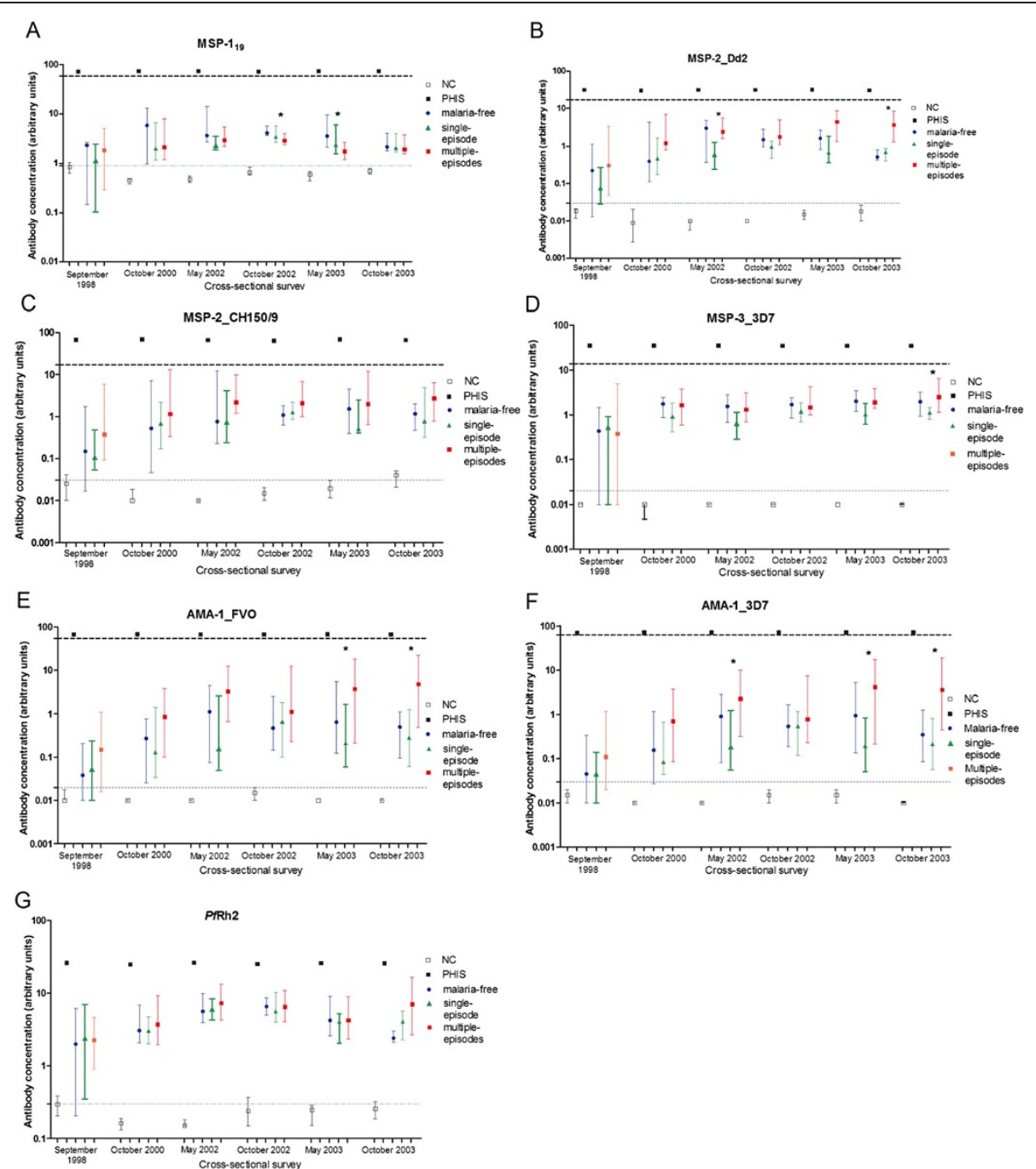

Figure 5 Distribution of antibody titers to individual merozoite antigens among the three groups of children. The panels show the distribution of antibody titers (median and interquartile range) in the malaria-free (blue circles), single-episode (green triangles) and multiple-episodes (red squares) groups of children at six cross-sectional surveys for the respective antigens: A) MSP-1 19, B) MSP-2_Dd2, C) MSP-2_CH150/9, D) MSP-3_3D7, E) AMA-1_FVO, F) AMA-1_3D7 and G) PfRh2. 'NC' refers to antibody titers in sera from P. falciparum-naïve adults (used here as negative controls). 'PHIS' refers to antibody titers in a pool of hyperimmune sera (used here as a positive control). The black bold dotted line shows the 'threshold' antibody concentrations to respective antigens that were calculated as described in the Results section. The thin dotted blue line shows the 'seropositivity cut off' based on the mean plus two standard deviations of the antibody titer obtained with the negative control sera.

the transient peaks in antibody titers are generated by the differentiation of naive B-cells into short-lived plasma cells (SLPCs) driven by concurrent infection rather than by long-lived plasma cells (LLPCs) generated from previous infections. Indeed, acute malaria infections in children lead to expansion of anti-merozoite antibodies and memory B-cell (MBC) pools which decline in the absence of parasites [35]. The dependence of antibody production in children on SLPCs may explain why antibody titers in the multiple episodes group were not higher than what we observed given the higher frequency of $P$. falciparum infections. Considering that differentiation of MBCs into SLPCs peaks six to eight days after re-exposure to antigen [36], antibody generation following acute infection in children may not be fast enough to prevent the rapid increase in parasitemia and thus clinical malaria which occurs approximately three days after blood-stage infection [37]. The incidence of malaria in children may thus be a reflection of the intensity of parasite exposure in individuals whose LLPCs are insufficiently developed to sustain antibodies at high enough concentrations to confer protection against malaria. However, given that the relative contributions of each $m s p 2$ genotype to the infection could not be determined using capillary sequencing as employed here, interpretation on disease causation and/or immunity should be cautious. 
Table 2 Temporal change in antibody titers among the three groups of children

\begin{tabular}{|c|c|c|c|c|c|}
\hline \multirow[t]{2}{*}{ Antigen } & \multirow[t]{2}{*}{ Group of children } & \multicolumn{4}{|l|}{ Coefficient $(95 \% \mathrm{Cl})$} \\
\hline & & Unadjusted & Adjusted $^{a}$ & Adjusted $^{b}$ & Adjusted $^{c}$ \\
\hline \multirow[t]{4}{*}{ MSP-1 19} & Malaria-free & Reference group & - & - & - \\
\hline & Single-episode & $-2.00(-11.27-7.27)$ & $-2.68(-12.03-6.74)$ & $-1.93(-10.32-6.46)$ & $-2.58(-11.10-5.93)$ \\
\hline & Multiple-episodes & $-2.54(-11.37-6.28)$ & $-2.54(-12.91-5.59)$ & $-3.82(-11.85-4.20)$ & $-4.93(-13.34-3.49)$ \\
\hline & $\begin{array}{l}\text { Single-episode versus } \\
\text { Multiple-episodes }\end{array}$ & $0.54(-5.88-6.96)$ & $0.99(-5.52-7.50)$ & $1.89(-4.02-7.80)$ & $2.35(-4.02-7.80)$ \\
\hline \multirow[t]{4}{*}{ MSP-2_Dd2 } & Malaria-free & Reference group & - & - & - \\
\hline & Single-episode & $0.43(-5.01-5.86)$ & $0.29(-5.27-5.864)$ & $0.24(-4.28-5.66)$ & $0.19(-5.17-5.55)$ \\
\hline & Multiple-episodes & $1.90(-3.01-7.35)$ & $1.90(-3.55-7.36)$ & $1.33(-3.69-6.36)$ & $0.94(-4.34-6.23)$ \\
\hline & $\begin{array}{l}\text { Single-episode versus } \\
\text { Multiple-episodes }\end{array}$ & $-1.74(-5.53-2.06)$ & $-1.62(-5.50-2.26)$ & $-0.93(-4.65-2.79)$ & $-0.75(-4.55-3.05)$ \\
\hline \multirow[t]{4}{*}{ MSP-2_CH150/9 } & Malaria-free & Reference group & & & \\
\hline & Single-episode & $0.24(-9.37-9.86)$ & $0.35(-10.21-9.50)$ & $0.41(-8.77-9.59)$ & $0.34(-9.72-9.05)$ \\
\hline & Multiple-episodes & $6.75(-2.39-15.89)$ & $5.83(-3.87-15.53)$ & $3.87(-4.91-12.66)$ & $2.68(-6.62-11.99)$ \\
\hline & $\begin{array}{l}\text { Single-episode versus } \\
\text { Multiple-episodes }\end{array}$ & $-6.51(-13.00-0.02)$ & $-6.18(-12.79-0.43)$ & $-3.46(-12.79-0.43)$ & $-3.02(-9.47-3.42)$ \\
\hline \multirow[t]{4}{*}{ MSP-3_3D7 } & Malaria-free & Reference group & - & - & - \\
\hline & Single-episode & $-2.42(-7.17-2.31)$ & $-2.23(-7.10-2.63)$ & $-2.37(-7.04-2.30)$ & $-2.37(-7.03-2.55)$ \\
\hline & Multiple-episodes & $-0.50(-5.02-4.02)$ & $-0.20(-4.98-4.57)$ & $-1.20(-5.67-3.26)$ & $-0.99(-5.72-3.72)$ \\
\hline & $\begin{array}{l}\text { Single-episode versus } \\
\text { Multiple-episodes }\end{array}$ & $-1.93(-5.24-1.38)$ & $-2.03(-5.43-1.37)$ & $-1.17(-4.48-2.15)$ & $-1.24(-4.64-2.17)$ \\
\hline \multirow[t]{4}{*}{ AMA-1_FVO } & Malaria-free & Reference group & - & - & - \\
\hline & Single-episode & $-3.71(-12.55-5.14)$ & $-4.32(-13.32-4.68)$ & $-3.64(-12.48-5.20)$ & $-3.76(-13.24-4.72)$ \\
\hline & Multiple-episodes & $3.87(-5.54-12.30)$ & $2.87(-5.97-11.71)$ & $3.10(-5.35-11.56)$ & $3.10(-6.82-10.94)$ \\
\hline & $\begin{array}{l}\text { Single-episode versus } \\
\text { Multiple-episodes }\end{array}$ & $-7.59(-13.71--1.47)^{*}$ & $-7.19(-13.40--0.98)^{*}$ & $-6.75(-12.97--0.52)^{*}$ & $-6.32(-12.64--0.01)^{*}$ \\
\hline \multirow[t]{4}{*}{ AMA-1_3D7 } & Malaria-free & Reference group & & & \\
\hline & Single-episode & $-2.83(-10.37-4.71)$ & $-3.43(-11.09-4.23)$ & $-2.79(-10.30-4.72)$ & $-3.37(-10.99-4.25)$ \\
\hline & Multiple-episodes & $3.17(-4.02-10.35)$ & $2.17(-5.36-9.70)$ & $2.83(-4.34-10.01)$ & $1.85(-5.68-9.38)$ \\
\hline & $\begin{array}{l}\text { Single-episode versus } \\
\text { Multiple-episodes }\end{array}$ & $-5.99(-11.22-0.77)^{*}$ & $-5.60(-10.90--0.31)^{*}$ & $-5.63(-10.92--0.33)^{*}$ & $-5.22(-10.60-1.52)$ \\
\hline \multirow[t]{4}{*}{ PfRh2 } & Malaria-free & Reference group & - & - & - \\
\hline & Single-episode & $-5.50(-13.21-2.27)$ & $-5.79(-13.71-2.12)$ & $-5.42(-13.22-2.38)$ & $-5.77(-13.74-2.19)$ \\
\hline & Multiple-episodes & $-1.72(-9.09-5.64)$ & $-2.25(-10.03-5.54)$ & $-1.99(-9.44-5.48)$ & $-2.57(-10.46-5.32)$ \\
\hline & $\begin{array}{l}\text { Single-episode versus } \\
\text { Multiple-episodes }\end{array}$ & $-3.74(-9.03-1.54)$ & $-3.55(-8.92-1.83)$ & $-3.44(-8.86-1.99)$ & $-3.21(-8.73-2.32)$ \\
\hline
\end{tabular}

${ }^{a}$ Adjusted for sickle cell trait (hemoglobin AS). ${ }^{b}$ Adjusted for whether a child was parasitemic or aparasitemic by microscopy at each of the six cross-sectional surveys 'Adjusted for sickle cell trait and whether a child was parasitemic or aparasitemic by microscopy at each of the six cross-sectional surveys. The coefficients indicate differences in the rates of change of antibody titers comparing the single- and multiple-episodes groups to the malaria-free group. Antibodies to AMA1_FVO increased significantly over five years when comparing the multiple- to the single-episode group. For all other antigens, the rates of change in antibody titers were not significant during this period. $\mathrm{Cl}$, confidence interval. ${ }^{*} \mathbf{P}<\mathbf{0 . 0 5}$.

The observed difference in the rates of change of antibody titers to AMA-1-FVO, but not to the other antigens in the three study groups, may be a reflection of antigen-specific differences in human immune responses to different antigens. Antibody longevity [38] and affinity [39] are known to vary between individual merozoite antigens. Nonetheless, the observed difference in the rates of change of antibody titers to AMA-1-FVO raises the question as to whether antibodies to AMA-1 are a good correlate of exposure [40] or of protective immunity [32].

We observed that children in the multiple-episodes group had more genetically-diverse infections compared to children in the single-episode and malaria-free groups. This finding is consistent with previous observations that 
genetically-diverse infections in children are associated with an increased risk of malaria [15,41,42]. We have previously shown that genetically-diverse infections are more often present in young children who develop severe non-cerebral malaria compared to age- and locationmatched children [43]. Taken together with the antibody data presented here, these findings suggest that the genetic diversity of asymptomatic infections in young children is a marker of the intensity of exposure to the parasite at a time when anti-merozoite antibodies have not attained concentrations required for protection against malaria.

In our study, other than at cross-sectional surveys, children were only tested for malaria parasites when they were symptomatic. Thus, it is possible that some asymptomatic infections were undetected, but that would apply equally to all groups and thus is unlikely to introduce bias. Another limitation of the study was the availability of antibody measurements only at the cross-sectional surveys and not when children were symptomatic. Thus, we could not compare antibody levels at the point of symptomatic infections in the multiple- and single-episode groups. We were also unable to account for the possible effects of HIV, malnutrition and prenatal $P$. falciparum exposure on antibody titers in the children studied here [44].

\section{Conclusions}

In summary, our data shows that in the context of a low malaria transmission setting, multiple episodes of clinical malaria are more likely the consequence of increased exposure rather than failure to acquire immunity. We hypothesize that intensive exposure induces antibodies at protective concentrations, while little to modest exposure may manifest as multiple clinical infections with low levels of antibodies. Future studies on the determinants of increased susceptibility to clinical malaria in areas with high malaria transmission intensity will complement the data presented here and help to define the tipping point in malaria transmission intensity where exposure translates into protective immunity as opposed to increased susceptibility to disease. Our data have implications for interventions including vaccines that effectively lower but do not completely abolish malaria transmission intensity. These interventions may reduce malaria transmission intensity below the threshold necessary to induce protective immunity and thus drive increased susceptibility to clinical malaria.

\section{Additional file}

Additional file 1: Supplementary figures. Demonstrate 1) the temporal variation in cohort size and parasite prevalence, 2 ) the distribution of the number of $P$. falciparum msp2 genotypes in the three groups of children and 3) the distribution of antibody titres in age-matched children in the Ngerenya, Chonyi and Tanzania cohorts.

\section{Abbreviations}

AMA-1: apical membrane antigen 1; AUs: arbitrary units; LLPCs: long-lived plasma cells; MBCs: memory B-cells; MFI: mean fluorescent intensity; MIG: malaria immune globulin; MSP: Merozoite surface protein; PHIS: pool of hyperimmune sera; SLPCs: short-lived plasma cells.

\section{Competing interests}

Josea Rono is currently working and affiliated with E\&K Health Consulting. E\&K Health Consulting was not involved in any role in the funding, design, conduct or decision to publish the study. JR was previously affiliated with Novartis but is no longer employed there. The other authors declare that they have no competing interests.

\section{Authors' contributions}

$J R, A F, K M$ and FO designed the study. JR, FG, GK and LM conducted the experiments. JR analyzed the data with advice from JO, AO, KM and FO. KM designed the cohort studies. GN, JW and BK maintained the longitudinal cohort data. All authors contributed to writing the manuscript. All authors read and approved the final manuscript.

\section{Acknowledgements}

We thank the children and their parents/guardians for their participation in the study. We are grateful to Kevin Tetteh for provision of MSP-1 19, MSP-2 and MSP-3 recombinant antigens. We thank Edmond Remarque and James Beeson for the generous provision of AMA-1 and Rh2 recombinant antigens, respectively. We acknowledge Alister Craig for providing the purified malaria malaria-free globulin. We thank Tom Williams for provision of data on sickle cell trait. We are also grateful to Barnes Kitsao and Moses Mosobo for help with data management. We acknowledge the support of the Kenya Medical Research Institute (KEMRI). This work is published with the permission of the director of KEMRI. This work was supported by a Wellcome Trust grant awarded to FHAO (grant number 089833). JR is supported by a Wellcome Trust PhD grant (grant number 084538).

\section{Author details}

${ }^{1}$ KEMRI-Wellcome Trust Research Programme, Centre for Geographical Medicine Research-Coast, Kilifi, Kenya. ${ }^{2}$ Infectious Diseases Unit, Department of Medicine, Solna, Karolinska Institutet, Stockholm, Sweden. ${ }^{3}$ Centre for Clinical Vaccinology and Tropical Medicine, Churchill Hospital, University of Oxford, Oxford, UK.

Received: 24 January 2015 Accepted: 27 April 2015

Published online: 13 May 2015

\section{References}

1. Greenwood BM. The microepidemiology of malaria and its importance to malaria control. Trans R Soc Trop Med Hyg. 1989;83:25-9.

2. Trape JF, Pison G, Spiegel A, Enel C, Rogier C. Combating malaria in Africa. Trends Parasitol. 2002;18:224-30.

3. Clark TD, Greenhouse B, Njama-Meya D, Nzarubara B, Maiteki-Sebuguzi C, Staedke SG, et al. Factors determining the heterogeneity of malaria incidence in children in Kampala. Uganda J Infect Dis. 2008;198:393-400.

4. Bejon P, Williams TN, Liljander A, Noor AM, Wambua J, Ogada E, et al. Stable and unstable malaria hotspots in longitudinal cohort studies in Kenya. PLoS Med. 2010;7:e1000304.

5. Mwangi TW, Fegan G, Williams TN, Kinyanjui SM, Snow RW, Marsh K. Evidence for over-dispersion in the distribution of clinical malaria episodes in children. PLoS One. 2008;3:e2196.

6. Smith DL, Dushoff J, Snow RW, Hay SI. The entomological inoculation rate and Plasmodium falciparum infection in African children. Nature. 2005:438:492-5.

7. Woolhouse ME, Dye C, Etard JF, Smith T, Charlwood JD, Garnett GP, et al. Heterogeneities in the transmission of infectious agents: implications for the design of control programs. Proc Natl Acad Sci U S A. 1997;94:338-42.

8. Kreuels B, Kobbe R, Adjei S, Kreuzberg C, von Reden C, Bater K, et al. Spatial variation of malaria incidence in young children from a geographically homogeneous area with high endemicity. J Infect Dis. 2008;197:85-93.

9. Bousema T, Drakeley C, Gesase S, Hashim R, Magesa S, Mosha F, et al. Identification of hot spots of malaria transmission for targeted malaria control. J Infect Dis. 2010;201:1764-74. 
10. Midega JT, Smith DL, Olotu A, Mwangangi JM, Nzovu JG, Wambua J, et al. Wind direction and proximity to larval sites determines malaria risk in Kilifi District in Kenya. Nat Commun. 2012;3:674.

11. Trape JF, Lefebvre-Zante E, Legros F, Ndiaye G, Bouganali H, Druilhe P, et al. Vector density gradients and the epidemiology of urban malaria in Dakar. Senegal Am J Trop Med Hyg. 1992;47:181-9.

12. Mwangi TW, Ross A, Snow RW, Marsh K. Case definitions of clinical malaria under different transmission conditions in Kilifi District. Kenya J Infect Dis. 2005;191:1932-9.

13. Polley SD, Conway DJ, Cavanagh DR, McBride JS, Lowe BS, Williams TN, et al. High levels of serum antibodies to merozoite surface protein 2 of Plasmodium falciparum are associated with reduced risk of clinical malaria in coastal Kenya. Vaccine. 2006;24:4233-46.

14. Murungi LM, Kamuyu G, Lowe B, Bejon P, Theisen M, Kinyanjui SM, et al. A threshold concentration of anti-merozoite antibodies is required for protection from clinical episodes of malaria. Vaccine. 2013;31:3936-42.

15. Rono J, Osier FH, Olsson D, Montgomery S, Mhoja L, Rooth I, et al. Breadth of anti-merozoite antibody responses is associated with the genetic diversity of asymptomatic Plasmodium falciparum infections and protection against clinical malaria. Clin Infect Dis. 2013;57:1409-16.

16. Olotu A, Fegan G, Wambua J, Nyangweso G, Ogada E, Drakeley C, et al. Estimating individual exposure to malaria using local prevalence of malaria infection in the field. PLoS One. 2012:7:e32929.

17. Liljander A, Wiklund L, Falk N, Kweku M, Martensson A, Felger I, et al. Optimization and validation of multi-coloured capillary electrophoresis for genotyping of Plasmodium falciparum merozoite surface proteins (msp1 and 2). Malar J. 2009:8:78.

18. Burghaus PA, Holder AA. Expression of the 19-kilodalton carboxy-terminal fragment of the Plasmodium falciparum merozoite surface protein-1 in Escherichia coli as a correctly folded protein. Mol Biochem Parasitol. 1994;64:165-9.

19. Reiling L, Richards JS, Fowkes FJ, Barry AE, Triglia T, Chokejindachai W, et al Evidence that the erythrocyte invasion ligand PfRh2 is a target of protective immunity against Plasmodium falciparum malaria. J Immunol. 2010;185:6157-67.

20. Taylor RR, Smith DB, Robinson VJ, McBride JS, Riley EM. Human antibody response to Plasmodium falciparum merozoite surface protein 2 is serogroup specific and predominantly of the immunoglobulin G3 subclass. Infect Immun. 1995;63:4382-8.

21. Polley SD, Tetteh KK, Lloyd JM, Akpogheneta OJ, Greenwood BM, Bojang KA, et al. Plasmodium falciparum merozoite surface protein 3 is a target of allelespecific immunity and alleles are maintained by natural selection. J Infect Dis. 2007:195:279-87.

22. Kocken $\mathrm{CH}$, Withers-Martinez C, Dubbeld MA, van der Wel A, Hackett F, Valderrama A, et al. High-level expression of the malaria blood-stage vaccine candidate Plasmodium falciparum apical membrane antigen and induction of antibodies that inhibit erythrocyte invasion. Infect Immun. 2002;70:4471-6.

23. Taylor TE, Molyneux ME, Wirima JJ, Borgstein A, Goldring JD, Hommel M. Intravenous immunoglobulin in the treatment of paediatric cerebral malaria. Clin Exp Immunol. 1992;90:357-62.

24. Bereczky S, Montgomery SM, Troye-Blomberg M, Rooth I, Shaw MA, Farnert A. Elevated anti-malarial lgE in asymptomatic individuals is associated with reduced risk for subsequent clinical malaria. Int J Parasitol. 2004;34:935-42.

25. Hay SI, Smith DL, Snow RW. Measuring malaria endemicity from intense to interrupted transmission. Lancet Infect Dis. 2008;8:369-78.

26. Noor AM, Gething PW, Alegana VA, Patil AP, Hay SI, Muchiri E, et al. The risks of malaria infection in Kenya in 2009. BMC Infect Dis. 2009;9:180.

27. Smith DL, Guerra CA, Snow RW, Hay SI. Standardizing estimates of the Plasmodium falciparum parasite rate. Malar J. 2007:6:131.

28. Smith DL, Hay SI, Noor AM, Snow RW. Predicting changing malaria risk after expanded insecticide-treated net coverage in Africa. Trends Parasitol. 2009;25:511-6

29. Rabe-Hesketch A. Multilevel and longitudinal modelling using STATA. London: STATA Corp; 2005. https://gllamm.org/handout_2012.pdf.

30. Jagannathan P, Muhindo MK, Kakuru A, Arinaitwe E, Greenhouse B, Tappero J, et al. Increasing incidence of malaria in children despite insecticide-treated bed nets and prompt anti-malarial therapy in Tororo. Uganda Malar J. 2012;11:435

31. Hudson Keenihan SN, Ratiwayanto S, Soebianto S, Krisin, Marwoto H, Krishnegowda G, et al. Age-dependent impairment of IgG responses to glycosylphosphatidylinositol with equal exposure to Plasmodium falciparum among Javanese migrants to Papua, Indonesia. Am J Trop Med Hyg. 2003;69:36-41.

32. Osier FH, Fegan G, Polley SD, Murungi L, Verra F, Tetteh KK, et al. Breadth and magnitude of antibody responses to multiple Plasmodium falciparum merozoite antigens are associated with protection from clinical malaria. Infect Immun. 2008;76:2240-8.

33. Osier FH, Murungi LM, Fegan G, Tuju J, Tetteh KK, Bull PC, et al. Allelespecific antibodies to Plasmodium falciparum merozoite surface protein-2 and protection against clinical malaria. Parasite Immunol. 2010;32:193-201.

34. Osier FH, Weedall GD, Verra F, Murungi L, Tetteh KK, Bull P, et al. Allelic diversity and naturally acquired allele-specific antibody responses to Plasmodium falciparum apical membrane antigen 1 in Kenya. Infect Immun. 2010;78:4625-33.

35. Weiss GE, Traore B, Kayentao K, Ongoiba A, Doumbo S, Doumtabe D, et al. The Plasmodium falciparum-specific human memory B cell compartment expands gradually with repeated malaria infections. PLoS Pathog. 2010;6:e1000912

36. Bernasconi NL, Traggiai E, Lanzavecchia A. Maintenance of serological memory by polyclonal activation of human memory B cells. Science. 2002;298:2199-202.

37. Simpson JA, Aarons L, Collins WE, Jeffery GM, White NJ. Population dynamics of untreated Plasmodium falciparum malaria within the adult human host during the expansion phase of the infection. Parasitology. 2002;124:247-63.

38. Akpogheneta OJ, Duah NO, Tetteh KK, Dunyo S, Lanar DE, Pinder M, et al. Duration of naturally acquired antibody responses to blood-stage Plasmodium falciparum is age dependent and antigen specific. Infect Immun. 2008;76:1748-55.

39. Reddy SB, Anders RF, Beeson JG, Farnert A, Kironde F, Berenzon SK, et al. High affinity antibodies to Plasmodium falciparum merozoite antigens are associated with protection from malaria. PLoS One. 2012;7:e32242.

40. Reiling L, Richards JS, Fowkes FJ, Wilson DW, Chokejindachai W, Barry AE, et al. The Plasmodium falciparum erythrocyte invasion ligand Pfrh4 as a target of functional and protective human antibodies against malaria. PLoS One. 2012;7:e45253.

41. Felger I, Irion A, Steiger S, Beck HP. Genotypes of merozoite surface protein 2 of Plasmodium falciparum in Tanzania. Trans R Soc Trop Med Hyg. 1999;93:3-9.

42. Mueller I, Schoepflin S, Smith TA, Benton KL, Bretscher MT, Lin E, et al. Force of infection is key to understanding the epidemiology of Plasmodium falciparum malaria in Papua New Guinean children. Proc Natl Acad Sci U S A. 2012;109:10030-5.

43. Lundblom K, Murungi L, Nyaga V, Olsson D, Rono J, Osier F, et al. Plasmodium falciparum infection patterns since birth and risk of severe malaria: a nested case-control study in children on the coast of Kenya. PLoS One. 2013;8:e56032.

44. Malhotra I, Dent A, Mungai P, Wamachi A, Ouma JH, Narum DL, et al. Can prenatal malaria exposure produce an immune tolerant phenotype? A prospective birth cohort study in Kenya. PLoS Med. 2009;6:e1000116.

\section{Submit your next manuscript to BioMed Central and take full advantage of:}

- Convenient online submission

- Thorough peer review

- No space constraints or color figure charges

- Immediate publication on acceptance

- Inclusion in PubMed, CAS, Scopus and Google Scholar

- Research which is freely available for redistribution 